\title{
Surgery of Posterior Inferior Cerebellar Artery (PICA) Aneurysm
}

Ivan $\mathrm{Ng}$ and Julian Han

\subsection{Sign and Symptoms}

Posterior circulation aneurysms account for approximately $10 \%$ of all aneurysms, which affect $1-6 \%$ of the population [1,2]. Aneurysms of the posterior inferior cerebellar artery (PICA) are very rare, only $0.5-3 \%$ of all aneurysms [3]. Patients with PICA aneurysms usually present with subarachnoid hemorrhage, or they might have symptoms due to compression of the brainstem or lower cranial nerves. Microsurgical clipping of PICA aneurysms is difficult and very challenging due to the limited working space and its surrounding neurovascular structure, the brainstem and lower cranial nerves IX, X, XI, and XII, and very often the aneurysm is located very deep and far from the surgeon's view. Surgical maneuvers require moving around and sometimes in between cranial nerves. PICA aneurysms may vary widely within a range in terms of their complex anatomy, as a result either of their origin, branching out of the vertebral artery (VA), or their course along the lower cranial nerve. The PICA itself is usually of a small caliber, and aneurysms on it with a wide neck create a difficult situation with respect to clipping the aneurysm and preserving the PICA.

I. $\mathrm{Ng}(\bowtie) \cdot$ J. Han

National Neuroscience Institute (NNI),

Singapore, Singapore

e-mail: ivan.ng.h.b@singhealth.com.sg

\subsection{Investigation and Vascular Anatomy}

The VA branches out of the subclavian artery, ascends along the lateral aspect of the cervical vertebra through transverse processes, turns posterior of the lateral mass of $\mathrm{C} 1$, and enters the subarachnoid space between foramen magnum and ring of $\mathrm{C} 1$. The initial part of the intradural VA passes the superior-anterior to the first cervical roots, then crosses in front of the dentate ligament, also in front of the accessory nerve at the spinal part. The VA then ascends in front of the hypoglossal nerve to reach the medulla at its anterior surface, where it meets and unites with the contralateral VA to become the basilar artery at the junction of the medulla and pons.

The PICA is the VA's largest and most clinically significant branch. The PICA is very complex, sometimes long and tortuous, having a variable course and a wide area of supply compared to other branches of the cerebellar arteries. It supplies the medulla, cerebellar tonsil, inferior part of the vermis, and inferior surface of the cerebellar hemisphere. The PICA has five segments: anterior medullary (p1), lateral medullary (p2), tonsillomedullary (p3), telovelotonsillar (p4), and cortical (p5) segments (Fig. 19.1).

The first segment (p1) starts from the PICA's origin just in front of the medulla near the inferior olivary, then turns posteriorly through hypoglossal rootlets and continues on, then 


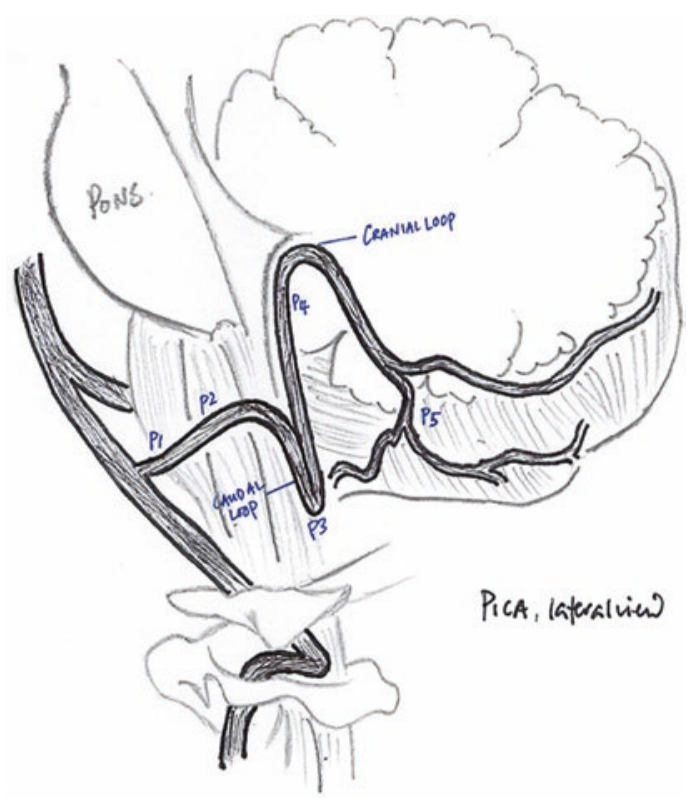

Fig. 19.1 Drawing of PICA and its segments: anterior medullary (p1), lateral medullary (p2), tonsillomedullary (p3), telovelotonsillar (p4), and cortical (p5) segments. It supplies the medulla, cerebellar tonsil, inferior vermis, and suboccipital surface of the cerebellar hemisphere

turning to the lateral surfaces of the medulla. The second segment (p2) starts from the turn at the prominent point of the olivary until the outlet of the lower cranial nerve. The third segment (p3) starts at the outlet of the lower cranial nerve and crosses medially at the posterior aspect of the medulla, making a big loop starting from the lateral surface of the tonsil until it reaches the pole and turning up at the medial surface of the tonsil. The fourth segment (p4) starts at the midpoint of the PICA at the medial surface of the tonsil, moves toward the fourth ventricle roof, then comes out through the fissure to reach the cerebellar surface. There is almost always a cranial loop where the apex usually overlies the inferior part of the medullary velum. The fifth segment (p5) starts where the PICA extends out from the fissure between the tonsil, vermis, and cerebellar hemisphere. It often happens that the bifurcation of the PICA occurs at this fifth segment; the lateral trunk will supply the tonsil and cerebral surface, while the medial trunk will supply the vermis.
CT angiography and 3D reconstruction are usually sufficient for diagnostic purposes and surgical planning. Interventional angiography is rarely required, but for selective cases it still provides additional value for treatment.

\subsection{Steps in Surgery}

Microsurgical treatment of PICA aneurysms could be done either by clipping the aneurysm or trapping/occluding it with bypass surgery.

PICA aneurysms most commonly occur at the p2 and p3 segments [4]. Common approaches include by the lateral suboccipital or far lateral suboccipital for more proximal PICA aneurysms and paramedian or midline suboccipital for more distal PICA aneurysms. Other more complex skull-base approaches have also been described, such as retro-labyrinthine presigmoid and transsigmoid exposures. A modified far lateral approach, which has been the approach most frequently used by the senior author in dealing with PICA aneurysms, is described here.

The patient may be placed in a true lateral position, with the operative side facing upward (Fig. 19.2). The dependent extremity is hung in a sling. The contralateral shoulder is pushed forward anteriorly, and there is mild inferior traction of the shoulder with tape. The vertex of the head is tilted slightly toward the ground, and there is also a slight lateral rotation of the head toward the ground. This opens up the working space at the lateral suboccipital region. Skin incision is made in the manner of golf club with the descending limb remaining at the midline from inion to C2. The muscles and soft tissue are dissected with monopolar blade then retracted, exposing the occipital, mastoid bones and posterolateral arch of $\mathrm{C} 1$; when dissection is done near the foramen magnum, especially $2-3 \mathrm{~cm}$ laterally from the midline, where yellowish fat tissue occurs, one must be careful not to injure the extracranial VA. Place a burr hole near the transverse-sigmoid junction, gently detach the dura from the bone, and perform a craniotomy up to $1 \mathrm{~cm}$ from the midline and the foramen magnum inferiorly; additional $\mathrm{C} 1$ laminectomy is necessary for better 

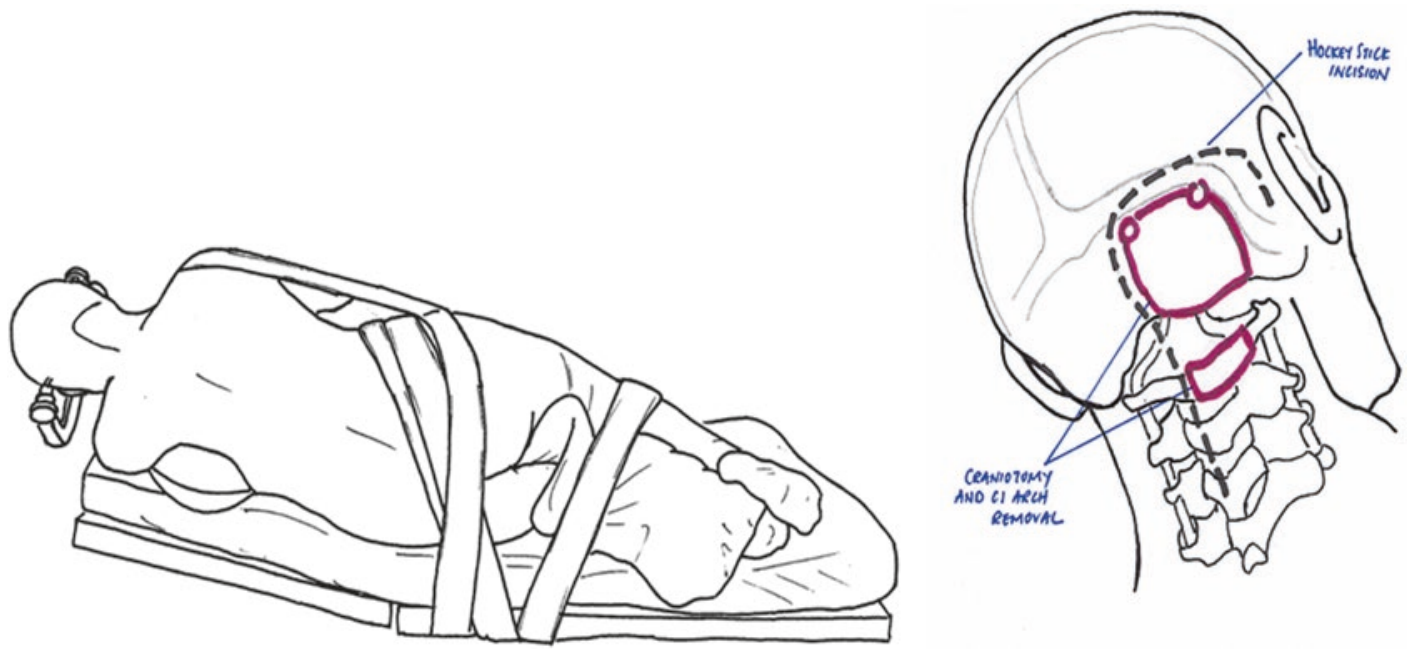

Fig. 19.2 Left: Patient is placed in lateral position with operative side upward. The dependent extremity is hung in a sling. The contralateral shoulder is pushed forward anteriorly, and there is mild inferior traction of shoulder with tape. Right: Vertex of head is tilted slightly toward ground, and there is also a slight lateral rotation of head toward ground proximal control. Lateral suboccipital bony exposure should be up to the line where the VA enters the dura. The dura opening is a gentle curve and the edges are holded with silk stiches. The Cerebrospinal fluid needs to be released slowly so the brain is relaxed, and everything must be done under a microscopic view. Preparing the vertebra artery (VA) for the proximal control and usually the VA is under the dentate ligament when it comes to intracranially. The ligament must be cut gently from the foramen magnum and at $\mathrm{C} 1$ to enable viewing of the VA anteriorly and to widen the surgical corridor by detaching the upper cervical spinal cord. The proximal VA just after it becomes intracranial should be prepared for temporary clipping. In cases where the PICA originates right at the point where the VA pierces the dura, extradural/extracranial dissection of the VA may be performed. Typically, authors expose the VA at the point where the vessel forms a groove in the upper surface of the $\mathrm{C} 1$ lamina, which is marked anatomically by the sulcus limitans. Dissection of the PICA should not be a difficult procedure; in fact, it is relatively simple, and one should be able to locate the aneurysm by simply following the PICA's course.
We can start following the PICA just under the tip of the tonsil and tracking it to the cistern. Sharp dissection may be done by opening the arachnoid layer to expose the vagoaccessory triangle, which provides a good natural working space, and many surgeons use this method in a far lateral approach (Fig. 19.3). Superiorly there is the vagus nerve, laterally the accessory nerve, and medially the medulla. One's view of the meeting point of the VA and PICA is usually obstructed by the IX-X-XI complex. Each case may have unique features, such as the shape of the aneurysm, the parent artery anatomy, and the tortuosity of the vessel, so the dissection strategy, including the pathway, should be formulated accordingly.

For more distally positioned aneurysms, surgical access may be achieved using a telovelar approach (Figs. 19.4 and 19.5).

PICA aneurysms are often associated with unusual anatomy, and direct clipping is not always possible. Several factors such as the branch anatomy, the size of the aneurysm, dolicho-ectatic morphology, or even atherosclerotic neck, will contribute to the difficulty. This then requires alternative techniques such as a trapping and 

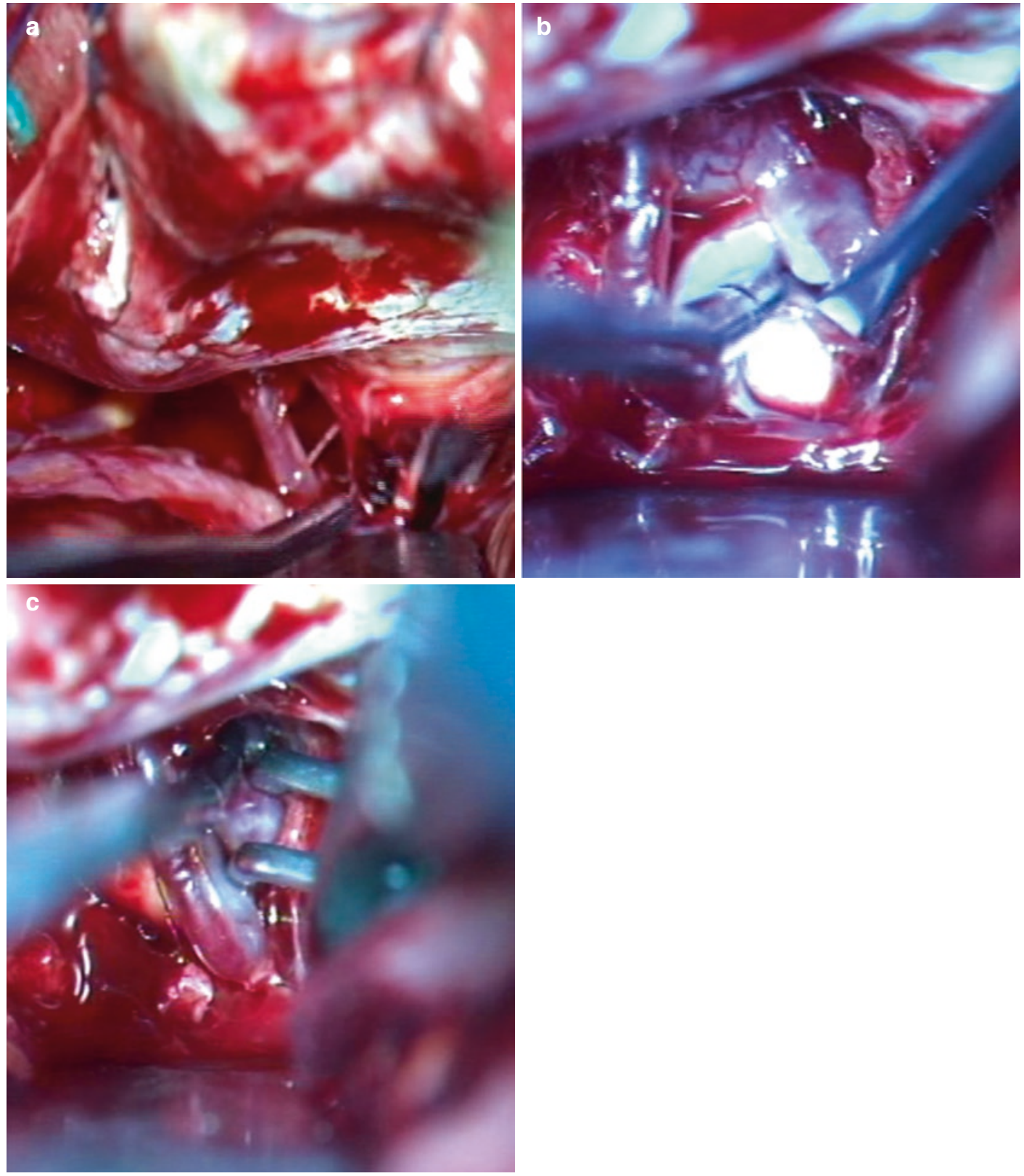

Fig. 19.3 (a) This patient has a proximal left PICA aneurysm in lateral medullary zone. The approach is via a modified far lateral approach. (b) The dissection is deep to

bypass procedure. Preoperative study and planning are very important if revascularization procedures are necessary. This is particularly significant in proximally located aneurysms, particularly at the VA-PICA junction. In scenarios potentially requiring the occlusion of the PICA, bypass procedures may be necessary. the rootlets of the accessory nerve to expose the aneurysm neck. (c) The aneurysm was clipped simply with a bayonet clip

There are various options of extra-intracranial (EC-IC) bypass surgery. However, Authors generally favor an in situ bypass (PICA-PICA) that matches donor and recipient arteries well because it can be performed quickly.

Bypass options include the following (Fig. 19.6): 

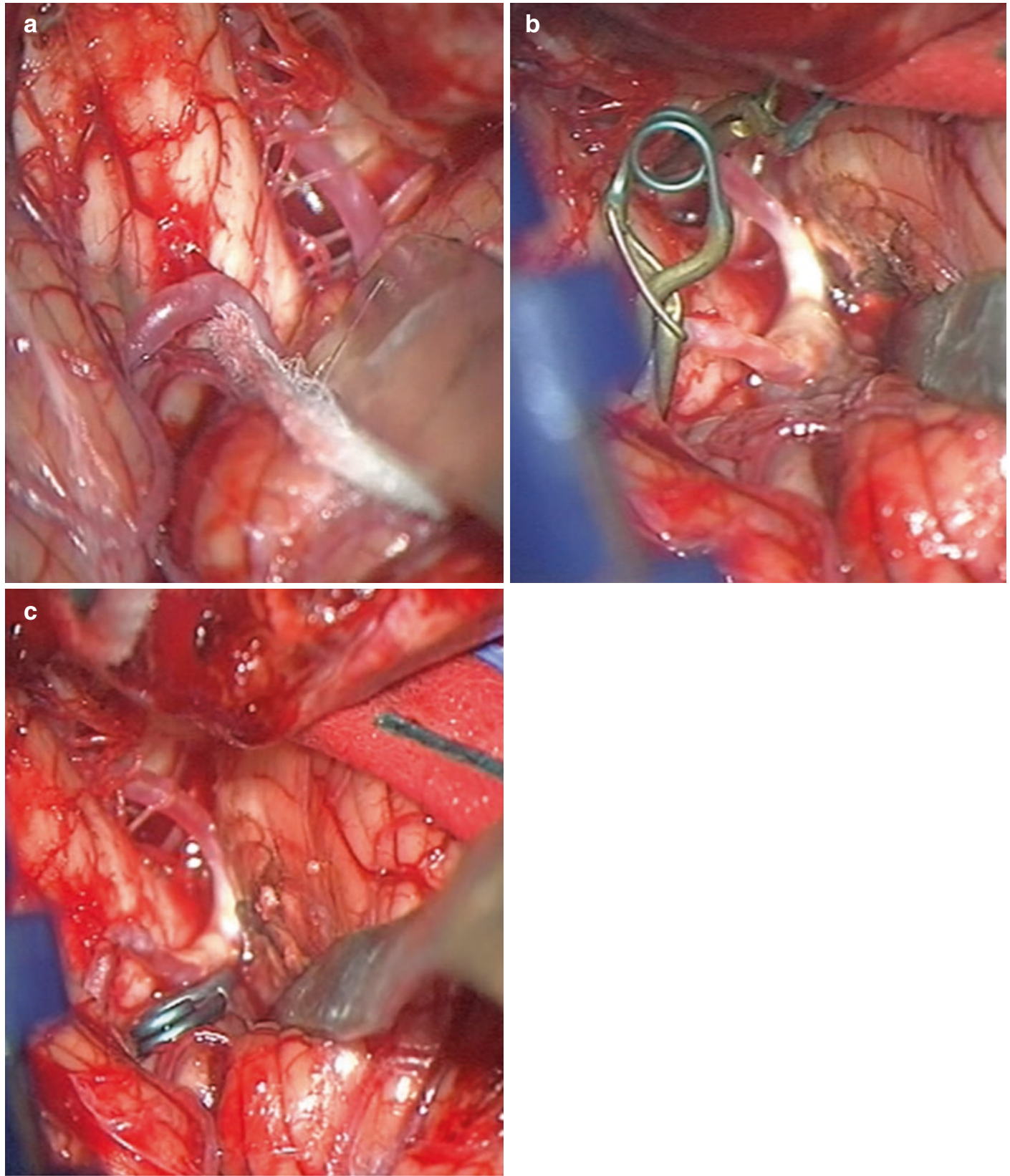

Fig. 19.4 (a) Some PICA aneurysms are located more distally, such as this one, at the ascending loop of the p3 segment of the left PICA. This is better accessed via a midline suboccipital approach. (b) Proximal and distal

1. PICA-to-PICA bypass,

2. Reimplantation of PICA in VA,

3. Reanastomosis of PICA,

4. High-flow bypass from VA to PICA using radial artery graft,

5. Occipital artery (OA)-PICA bypass. control was achieved with temporary clipping, before dissecting out the aneurysm neck. (c) The aneurysm was clipped simply with a straight clip

PICA-PICA bypass involves the using two PICAs that lie close to each other at the midline in the ascending portion of the $\mathrm{p} 3$ segment running at the medial surface of the tonsil. The two loops are generously mobilized and their arachnoid trabecula is cut to make the vessel free 

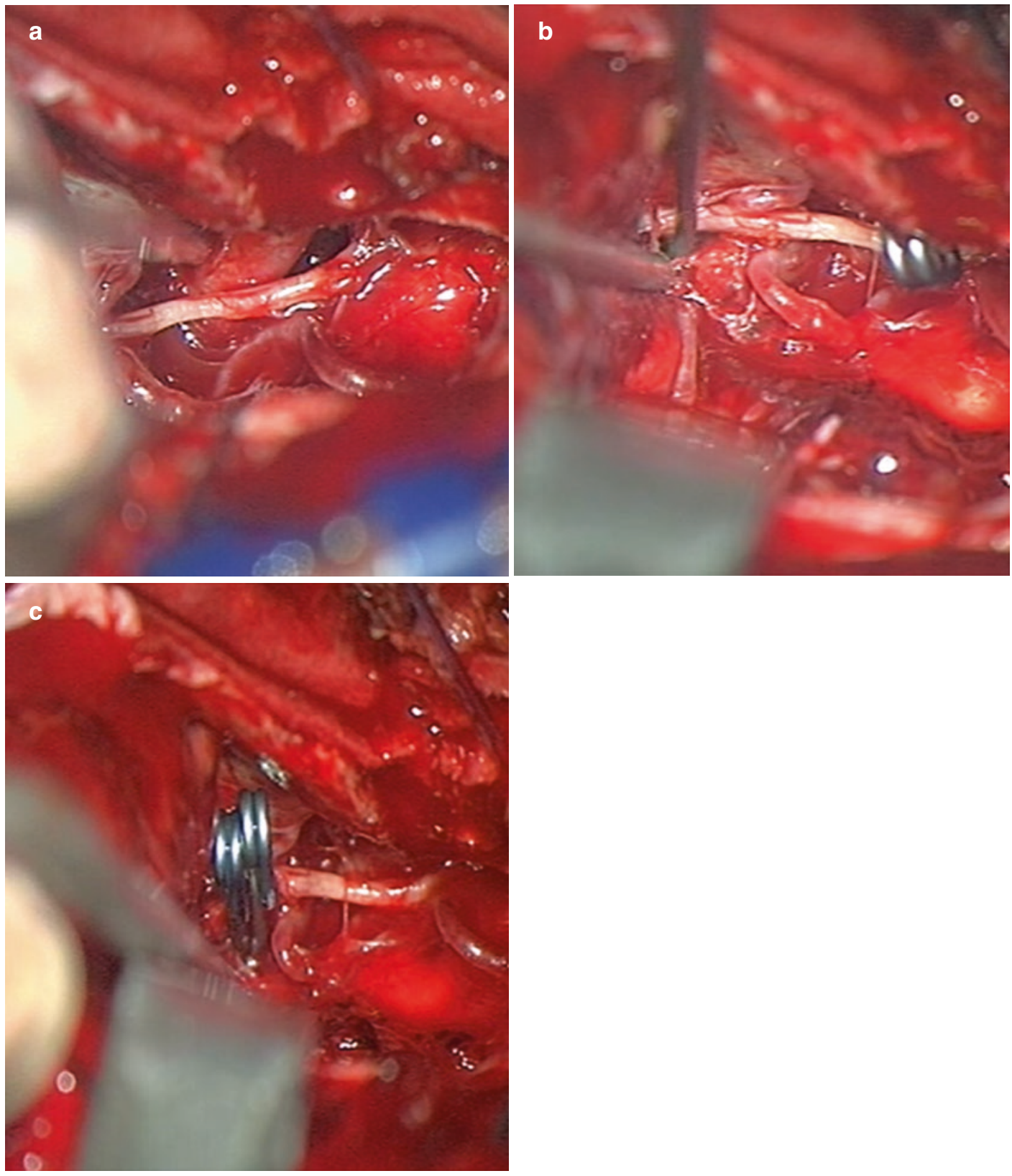

Fig. 19.5 (a) This is a case of proximal right PICA aneurysm located at the lateral medullary segment. Dissection was via a modified far lateral approach. Intradural VA was

and to allow for tension-free side-to-side anastomosis. With the temporary clips in place on both PICAs, a longitudinal arteriotomy is made in each artery, ideally two to three times the PICA diameter. Both PICAs should have almost identical diameters, or the donor should be larger than the ipsilateral recipient. identified for proximal control. (b) Aneurysm neck excised just beyond the traversing accessory nerve. (c) The aneurysm neck was obliterated with tandem clipping

Reimplantation of the PICA can be done if the clip obliteration of the aneurysm also sacrifices the PICA but preserves the VA. The occluded PICA can be revascularized by cutting at its origin, transposing and reimplanting it proximally to the original PICA origin. This is an end-to-side anastomosis. Reimplantation is probably the 

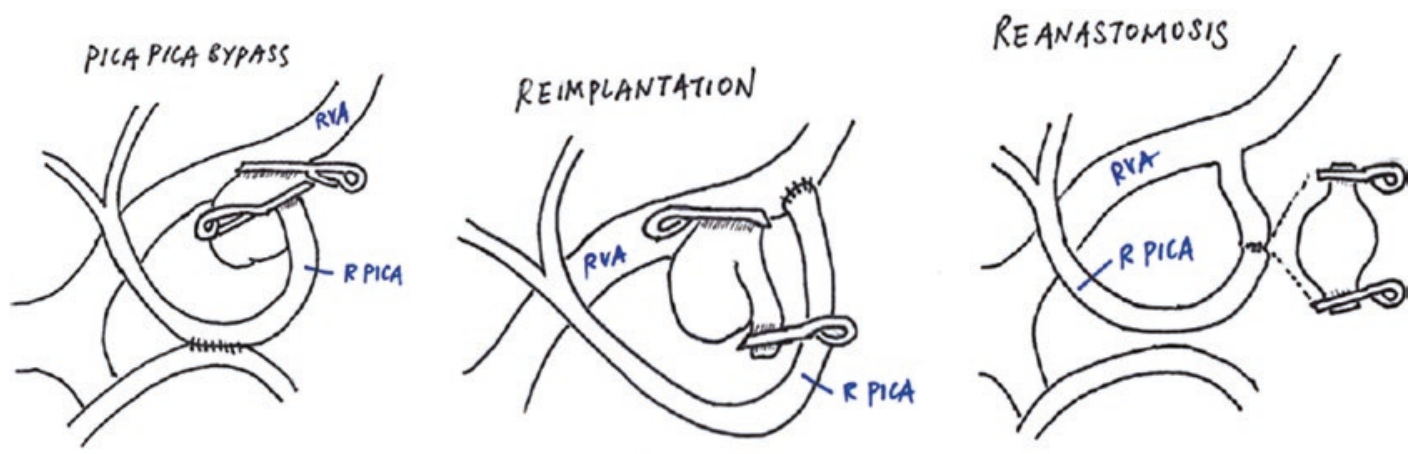

Fig. 19.6 Drawing of PICA-PICA bypass (left), reimplantation of PICA to VA (middle), and reanastomosis of PICA (right)

hardest of the IC-IC bypasses because the location is deep and it is surrounded by all the lower cranial nerves. The complication of postoperative lower cranial nerve palsy is much higher with this surgery.

Reanastomosis involves reconnecting the transected ends of a parent artery after excising the aneurysm; it is an end-to-end anastomosis. The afferent and efferent ends are generously mobilized from their arachnoid adhesions to allow approximation without tension. A PICA's tortuosity usually provides adequate length to get to the arteries without tension. Ideal situations for such a bypass include aneurysms that are fusiform in their distal portions and have no branches. The end-to-end anastomosis line is shorter, making suturing quicker.

Large complex aneurysms at the VA-PICA junction may be trapped and reconstructed with an interposition graft using the radial artery. The donor site is usually at V3, and the recipient site is a PICA. A radial artery graft is more suitable in terms of caliber compared to a saphenous vein graft. Interposition means that two grafts are needed, with both requiring two anastomoses, either end to end or end to side.

An alternative bypass is an OA-PICA bypass for large complex aneurysms involving the VAPICA junction. The $\mathrm{OA}$ is located at the posteriormost of the eight branches of the External Carotid artery and lies medially and posteriorly of the mastoid process. It is identified preoperatively using a handheld Doppler probe. It is usually densely adhered to its surrounding muscular tissue, making it more difficult to dissect compared to the Superficial Temporal Artery. It is an endto-side anastomosis.

Parent artery sacrifice of the PICA is a possible treatment, but it may cause medullary and cerebellar infarcts that will be followed by malignant edema, causing acute hydrocephalus or, in the worst case, direct brainstem compression. These complications may be tolerable if the patient's condition deteriorates, in which case urgent decompressive craniectomy may become necessary. Many surgeons might simply sacrifice the parent artery without revascularization if the aneurysm arises at the most distal two segments of the PICA [5]. The more proximally parent PICA should not be sacrificed because of variations in perforating vessels that may arise from more proximal segments. Liew et al. have suggested that if an aneurysm is located distally to the last perforator artery, then a clip may be safely placed on the parent artery after the perforator artery [6]. If there is no evidence that the patient will be able to tolerate PICA sacrifice, the surgeon will need to prepare all alternatives, including direct clipping with or without bypass to preserve the PICA; additional endovascular coiling will likely be required to achieve complete aneurysm occlusion [7].

\subsection{Expert Opinion}

PICA aneurysms are relatively rare and pose significant challenges to neurosurgeons owing to their proximity to critical structures and their 
variable complex anatomy. However, they can be successfully managed microsurgically, and the neurosurgeon has a variety of options at her disposal that can be used aside from direct clipping, such as occlusion or trapping, with several bypass possibilities.

\section{References}

1. Schievink WI. Intracranial aneurysms. N Engl J Med. 1997;336:28-40.

2. Rinkel GJ, et al. Prevalence and risk of rupture of intracranial aneurysms: a systematic review. Stroke. 1998;29:251-6.
3. Hudgins RJ, et al. Aneurysms of the posterior inferior cerebellar artery. A clinical and anatomical analysis. J Neurosurg. 1983;58:381-7.

4. Rodríguez-Hernández A, et al. Distal aneurysms of intracranial arteries: application of numerical nomenclature, predilection for cerebellar arteries, and results of surgical management. World Neurosurg. 2013;80:103-12.

5. Heros RC. Posterior inferior cerebellar artery. Editorial. J Neurosurg. 2002;97:747-8.

6. Liew D, et al. Surgical management of ruptured and unruptured symptomatic posterior inferior cerebellar artery aneurysms. Br J Neurosurg. 2004;18:608-12.

7. Abla AA, et al. Intracranial-to-intracranial bypass for posterior inferior cerebellar artery aneurysms: options, technical challenges, and results in 35 patients. J Neurosurg. 2016;124(5):1275-86.

Open Access This chapter is licensed under the terms of the Creative Commons Attribution 4.0 International License (http://creativecommons.org/licenses/by/4.0/), which permits use, sharing, adaptation, distribution and reproduction in any medium or format, as long as you give appropriate credit to the original author(s) and the source, provide a link to the Creative Commons license and indicate if changes were made.

The images or other third party material in this chapter are included in the chapter's Creative Commons license, unless indicated otherwise in a credit line to the material. If material is not included in the chapter's Creative Commons license and your intended use is not permitted by statutory regulation or exceeds the permitted use, you will need to obtain permission directly from the copyright holder. 